POS PROCEEDINGS

\title{
Experimental news from a theoretical state: The "ppK
}

Eliane Epple ${ }^{10,9}$ for the HADES collaboration:

E-mail: eliane.epple@ph.tum.de

J. Adamczewski-Musch ${ }^{4}$, O. Arnold ${ }^{10,9}$, E.T. Atomssa ${ }^{15}$, C. Behnke ${ }^{8}$,

J.C. Berger-Chen ${ }^{10,9}$, J. Biernat ${ }^{3}$, A. Blanco ${ }^{2}$, C. Blume ${ }^{8}$, M. Böhmer ${ }^{10}$, P. Bordalo ${ }^{2}$, S. Chernenko ${ }^{7}$, C. Deveaux ${ }^{11}$, A. Dybczak ${ }^{3}$, L. Fabbietti ${ }^{10,9}$, O. Fateev ${ }^{7}$, P. Fonte ${ }^{2, a}$,

C. Franco ${ }^{2}$, J. Friese ${ }^{10}$, I. Fröhlich ${ }^{8}$, T. Galatyuk ${ }^{5, b}$, J. A. Garzón ${ }^{17}$, K. Gill ${ }^{8}$, M. Golubeva ${ }^{12}$, F. Guber ${ }^{12}$, M. Gumberidze ${ }^{5, b}$, S. Harabasz ${ }^{5,3}$, T. Hennino ${ }^{15}$,

S. Hlavac ${ }^{1}$, C. Höhne ${ }^{11}$, R. Holzmann ${ }^{4}$, A. lerusalimov ${ }^{7}$, A. Ivashkin ${ }^{12}$, M. Jurkovic ${ }^{10}$,

B. Kämpfer ${ }^{6, c}$, T. Karavicheva ${ }^{12}$, K. Kardan ${ }^{8}$, I. Koenig ${ }^{4}$, W. Koenig ${ }^{4}$, B. W. Kolb ${ }^{4}$,

G. Korcyl ${ }^{3}$, G. Kornakov ${ }^{5}$, R. Kotte ${ }^{6}$, A. Krása ${ }^{16}$, E. Krebs ${ }^{8}$, H. Kuc ${ }^{3,15}$, A. Kugler ${ }^{16}$, T. Kunz ${ }^{10}$, A. Kurepin ${ }^{12}$, A. Kurilkin ${ }^{7}$, P. Kurilkin ${ }^{7}$, V. Ladygin ${ }^{7}$, R. Lalik ${ }^{10,9}$, K. Lapidus ${ }^{10,9}$, A. Lebedev ${ }^{13}$, L. Lopes ${ }^{2}$, M. Lorenz ${ }^{8}$, T. Mahmoud ${ }^{11}$, L. Maier ${ }^{10}$, A. Mangiarotti ${ }^{2}$, J. Markert ${ }^{8}$, V. Metag ${ }^{11}$, J. Michel ${ }^{8}$, C. Müntz ${ }^{8}$, R. Münzer ${ }^{10,9}$, L. Naumann ${ }^{6}$, M. Palka ${ }^{3}$, Y. Parpottas ${ }^{14, d}$, V. Pechenov ${ }^{4}$, O. Pechenova ${ }^{8}$, V. Petousis ${ }^{14}$, J. Pietraszko ${ }^{4}$, W. Przygoda ${ }^{3}$, B. Ramstein ${ }^{15}$, L. Rehnisch ${ }^{8}$, A. Reshetin ${ }^{12}$, A. Rost $^{5}$, A. Rustamov ${ }^{8}$, A. Sadovsky ${ }^{12}$, P. Salabura ${ }^{3}$, T. Scheib ${ }^{8}$, K. Schmidt-Sommerfeld ${ }^{10}$, H. Schuldes ${ }^{8}$, P. Sellheim ${ }^{8}$, J. Siebenson ${ }^{10}$, L. Silva ${ }^{2}$, Yu.G. Sobolev ${ }^{16}$, S. Spataro ${ }^{e}$, H. Ströbele ${ }^{8}$, J. Stroth ${ }^{8,4}$, P. Strzempek ${ }^{3}$, C. Sturm ${ }^{4}$, O. Svoboda ${ }^{16}$, A. Tarantola ${ }^{8}$, K. Teilab ${ }^{8}$, P. Tlusty ${ }^{16}$, M. Traxler ${ }^{4}$, H. Tsertos ${ }^{14}$, T. Vasiliev ${ }^{7}$, V. Wagner ${ }^{16}$, C. Wendisch ${ }^{6, c}$, J. Wirth ${ }^{10,9}$, J. Wüstenfeld ${ }^{6}$, Y. Zanevsky ${ }^{7}$, P. Zumbruch ${ }^{4}$ 


POS PROCEEDINGS

${ }^{1}$ Institute of Physics, Slovak Academy of Sciences, 84228 Bratislava, Slovakia

${ }^{2}$ LIP-Laboratório de Instrumentação e Física Experimental de Partículas , 3004-516 Coimbra, Portugal

${ }^{3}$ Smoluchowski Institute of Physics, Jagiellonian University of Cracow, 30-059 Kraków, Poland

${ }^{4}$ GSI Helmholtzzentrum für Schwerionenforschung GmbH, 64291 Darmstadt, Germany

${ }^{5}$ Technische Universität Darmstadt, 64289 Darmstadt, Germany

${ }^{6}$ Institut für Strahlenphysik, Helmholtz-Zentrum Dresden-Rossendorf, 01314 Dresden, Germany

${ }^{7}$ Joint Institute of Nuclear Research, 141980 Dubna, Russia

${ }^{8}$ Institut für Kernphysik, Goethe-Universität, 60438 Frankfurt, Germany

${ }^{9}$ Excellence Cluster 'Origin and Structure of the Universe', 85748 Garching, Germany

${ }^{10}$ Physik Department E12, Technische Universität München, 85748 Garching, Germany

${ }^{11}$ II.Physikalisches Institut, Justus Liebig Universität Giessen, 35392 Giessen, Germany

${ }^{12}$ Institute for Nuclear Research, Russian Academy of Science, 117312 Moscow, Russia

${ }^{13}$ Institute of Theoretical and Experimental Physics, 117218 Moscow, Russia

${ }^{14}$ Department of Physics, University of Cyprus, 1678 Nicosia, Cyprus

${ }^{15}$ Institut de Physique Nucléaire (UMR 8608), CNRS/IN2P3 - Université Paris Sud, F-91406 Orsay Cedex, France

${ }^{16}$ Nuclear Physics Institute, Academy of Sciences of Czech Republic, 25068 Rez, Czech Republic

${ }^{17}$ LabCAF. F. Física, Univ. de Santiago de Compostela, 15706 Santiago de Compostela, Spain

${ }^{a}$ also at ISEC Coimbra, Coimbra, Portugal

${ }^{b}$ also at ExtreMe Matter Institute EMMI, 64291 Darmstadt, Germany

${ }^{c}$ also at Technische Universität Dresden, 01062 Dresden, Germany

${ }^{d}$ also at Frederick University, 1036 Nicosia, Cyprus

${ }^{e}$ also at Dipartimento di Fisica and INFN, Università di Torino, 10125 Torino, Italy

We present $\mathrm{p}+\mathrm{p}$ data, measured by the HADES spectrometer at $E_{\text {Kin }}$ (proton) $=3.5 \mathrm{GeV}$. We have analyzed the final state $p+p \rightarrow p \Lambda K^{+}$in view of a possible intermediate state $p+p \rightarrow \bar{K} N N+$ $K^{+}$. The $\bar{K} N N$ is the lightest candidate for a cluster of an anti-kaon bound to nucleons, and a current topic of theoretical and experimental interest. We have analyzed our data with a PartialWave Analysis that includes also the production of intermediate $\mathrm{N}^{*}$-resonances $\left(\rightarrow K^{+} \Lambda\right)$. The result of the fit describes the data well and a statistical test showed no major deviations between model and data which could be attributed to a production of a kaonic nuclear bound state. We have, thus, started to focus on the determination of an upper limit of its production cross section.

52 International Winter Meeting on Nuclear Physics - Bormio 2014,

27-31 January 2014

Bormio, Italy

(c) Copyright owned by the author(s) under the terms of the Creative Commons Attribution-NonCommercial-ShareAlike Licence 


\section{Anti-Kaonic Nuclear Bound States}

Bound states of anti-kaons and nucleons have been a constant topic of discussion since the early 60's, beginning from Y. Nogamis pioneering work [1-19]. While the early calculations studied all sorts of bound states throughout the periodic table, the recent theoretical and experimental interest is focused on light bound states such as $\bar{K} N N$ and $\bar{K} N N N$. The calculations of the properties of this state have evolved meanwhile to an advanced form, were the simplest cluster - the $\bar{K} N N$ - is calculated either in a Faddeev [20-28] or a variational approach [18, 19,29-35]. The predicted binding energies and widths in these approaches vary between $\mathrm{B}=10-100 \mathrm{MeV}$ and $\Gamma=40-110$ $\mathrm{MeV}$, see e.g. summary tables in Refs. [36,37], giving a broad range of possible properties.

The experimental efforts, on the other hand, presented three candidates, all of them heavily criticized [38-42]. Their properties are: $\mathrm{B}=115 \mathrm{MeV}$ and $\Gamma=67 \mathrm{MeV}$ [38], $\mathrm{B}=153 \mathrm{MeV}$ and $\Gamma<24$ $\mathrm{MeV}[39,40], \mathrm{B}=103 \mathrm{MeV}$ and $\Gamma=118 \mathrm{MeV}[41,42]$. These are contradicting results. The LEPS collaborations on the other hand recently published results from the reaction $\gamma+d \rightarrow X K^{+} \pi^{-}$in which no signal from the $\bar{K} N N(\hat{=} X)$ was observed and an upper limit for the production rate was reported [43]. The situation from theoretical and experimental side is inconclusive and, thus, demands more experimental data to resolve the issue of observable kaonic nuclear bound states.

\section{The p+p Experiment of HADES}

In 2007 the HADES detector ${ }^{1}$ [44] recorded $1.2 \cdot 10^{9}$ events from $\mathrm{p}+\mathrm{p}$ collisions induced by a proton beam with $E_{K i n}=3.5 \mathrm{GeV}$ impinged on a liquid hydrogen target. These events were analyzed in view of reaction $p+p \rightarrow p \Lambda K^{+}$. This final state is interesting, as it contains the decay products of one of the four decay channels ${ }^{2}$ of the $\bar{K} N N$ and could, thus, stem from the possible intermediate state $p+p \rightarrow \bar{K} N N+K^{+}$. A large contribution of a $\bar{K} N N$ with a narrow width would manifest itself as a bump in the $p \Lambda$ invariant mass spectrum. If the production yield or the $\Lambda p$ decay width is, however, small, the background description becomes more and more crucial to distinguish a possible signal from statistical fluctuations or background structures.

As the kinematics of the $p K^{+} \Lambda$ final state is rather complex due to the presence of intermediate $\mathrm{N}^{*}$ resonances $\left(\rightarrow K^{+} \Lambda\right)[45,46]$, the modeling of the event distributions is difficult. This is resolved with help of a partial wave analysis (PWA) framework from the Bonn-Gatchina group $[47,48]$. Besides the possibility to describe the complete event kinematics, this framework has the advantage that a production of a kaonic nuclear bound state can be implemented consistently with correct quantum numbers $\left(J^{P}=0^{-}\right)$. In this way, a possible interference between the cluster production and other intermediate states is taken into account.

\section{Data Selection}

The reaction $p+p \rightarrow p \Lambda K^{+}$has been selected in the following way. Two scenarios have been analyzed: I) in which all four charged particles $\left(\Lambda \rightarrow p \pi^{-}\right)$have been detected in the HADES

\footnotetext{
${ }^{1}$ High Acceptance Di-Electron Spectrometer, located at the GSI Helmholtzzentrum für Schwerionenforschung

${ }^{2} \rightarrow \Lambda N, \rightarrow \Sigma N, \rightarrow \Lambda N \pi, \rightarrow \Sigma N \pi$
} 
spectrometer; and II) in which the proton from the $\Lambda$ decay was detected in the Forward Wall ${ }^{3}$. These two data sets are named HADES and WALL, respectively. In both data sets, the protons and the pion were identified via their energy-loss in the MDC drift chambers via graphical cuts in the $\mathrm{dE} / \mathrm{dx}$ vs. momentum distribution. The particle detected in the Forward Wall can not be assigned with a PID as no underlying information is available. According to simulations, however, the hit in the forward wall is with a high probability $(\sim 90 \%)$ due to a proton and, thus, this assumption is used for the further analysis. The kaon was identified via its reconstructed time-of-flight which is used to calculate the particles mass. Kaons with a mass between 0-680 MeV/c ${ }^{2}$ (HADES) and 230-640 MeV/c ${ }^{2}$ (WALL) were accepted for the event selection. After the particle identification a kinematic fit of an exclusive $p \Lambda K^{+}$production is applied to the each selected event. The kinematic fit tests to which degree momentum and energy conservation are fulfilled by the four-vectors of the particles. Furthermore, it tests whether the invariant mass of the $\mathrm{p}$ and the $\pi^{-}$delivers a mass near the nominal $\Lambda$ mass of $1116 \mathrm{MeV} / \mathrm{c}^{2}$. Events with good fit quality were selected for further data analysis. This results in $\sim 1300$ events from the HADES statistic with a background of $\sim 7 \%$ and 9000 events from the WALL statistic with a background of $\sim 15 \%\left(K^{+}\right.$mis-identification and $p K^{+} \Sigma^{0}$-production). These selected events are then used as input for a partial wave analysis.

\section{The PWA Framework}

From these selected events the partial wave analysis uses the four-vectors from the three measured particles p, $\Lambda$, and $K^{+}$as well as phase space simulations as input. Both sets of four-vectors are reduced to the acceptance of the spectrometer, implying the advantage that errors due to an acceptance correction are avoided.

The transition from an initial state $(\mathrm{p}+\mathrm{p})$ into a final state $\left(p \Lambda K^{+}\right)$proceeds via many intermediate states. Each transition wave in the BG-PWA is parametrized by an amplitude $\left(a_{1}\right)$ a phase $\left(a_{2}\right)$ and an energy dependent strength $\left(a_{3}\right)$ according to:

$$
A_{t r}(s)=\left(a_{1}+a_{3} \sqrt{s}\right) \cdot e^{i a_{2}} .
$$

$A_{t r}(s)$ is a part of the full amplitude that is described by spin momentum operators which model the production and decay process dependent on the quantum numbers of the three-particle state $[47,48]$. As the center of mass energy of this experiment was constant, the energy dependent parameter $a_{3}$ was set to zero. The possible intermediate states are implemented as follows. All candidates of $\mathrm{N}^{*}$ resonances listed in the PDG that have an observed coupling to $K^{+} \Lambda$ and a mass between 1600 $2100 \mathrm{MeV} / \mathrm{c}^{2}$ are included as possible intermediate states. Furthermore, the production of $p K^{+} \Lambda$ can proceed via non-resonant formation, incorporating several angular momenta between the three particles. This leads to many possible transition waves that are grouped according to the total spin and parity $\left(J^{P}\right)$ of the intermediate state. All waves in the same spin-parity group can interfere as they stem from the same $p+p$ initial state wave. In the fitting process the kaonic cluster production was excluded to check how well the data are described by the already known standard sources.

Both data sets were fitted simultaneously on an event-by-event base by the sum of all transition amplitudes. The result of the fit are the values of the parameters $a_{1}$ and $a_{2}$ for each transition wave. Due to the many contributing processes, the final state observables can be described very

\footnotetext{
${ }^{3} \mathrm{~A}$ scintillator hodoscope $7 \mathrm{~m}$ downstream the target with a polar acceptance of $0.3^{\circ}$ to $7^{\circ}$.
} 

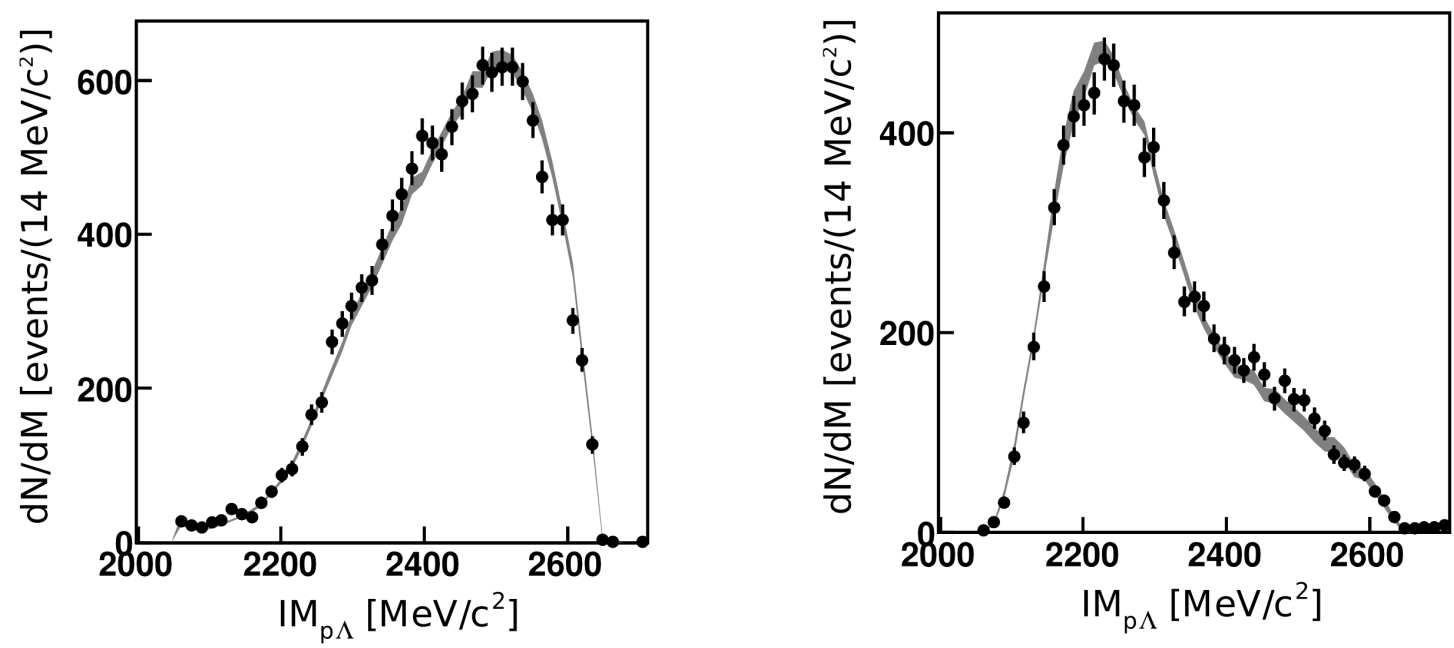

Figure 1: The experimental data in the HADES (left) and WALL (right) acceptance (black dots) compared to the four best out of 120 tested solutions of the PWA (gray band).

well by the interfered sum of all transition amplitudes. The fit is, however, not sensitive to the exact cocktail of transition waves implemented into the PWA. To evaluate this uncertainty several combinations of transition waves with varying contributions of $\mathrm{N}^{*}$ resonances and non resonant waves have been tested. In total 120 different solutions were fitted to the data and the results were categorized according to the minimum log-likelihood value of the fit. The four solutions with the best log-likelihood value were taken as a reference model for the data. Figure 1 shows how well these solutions (summarized as gray band) describe the experimental observable $I M_{p \Lambda}$.

\section{Cross Checks}

In order to check how a possible contribution of an intermediate state containing a kaonic nuclear cluster could influence or distort the fit result some cross checks have been performed. It is possible that an exclusion of an intermediate state with a kaonic cluster in the fit, whilst present in the experimental statistic, will lead to a wrong description of the data. This has been tested in the following way: First, events with a certain $p \Lambda$ invariant mass have been excluded from the fit and only the remaining statistic was fitted by the sum of all transition waves; then the new PWA solution was compared to the data in the whole mass range. A comparison of three fits: one including all data, one including only events outside the mass range $I M_{p \Lambda}=2200-2300 \mathrm{MeV} / \mathrm{c}^{2}$ and one including only events outside the mass range $I M_{p \Lambda}=2300-2400 \mathrm{MeV} / \mathrm{c}^{2}$, is presented in Figure 2. This figure shows the prediction of the three solutions in both acceptance ranges HADES and WALL. Here, only the HADES events were used for the fitting procedure to demonstrate the predictive power of the fit also for other acceptance regions. By comparing the three results one can validate how much the fit changes its prediction for a certain mass range when events inside of it are excluded. The right panels of Figure 2 show a zoom into the region in which a $\bar{K} N N$ signal could be located. No significant difference among these solutions is visible ${ }^{4}$, which indicates that a

\footnotetext{
${ }^{4}$ The lower right panel of Fig. 2 shows a slight deviation for low $M_{p \Lambda}$ masses for the green solution. This can, however, not be attributed to a signal bias as in this solution this mass range was explicitly included into the fit.
} 

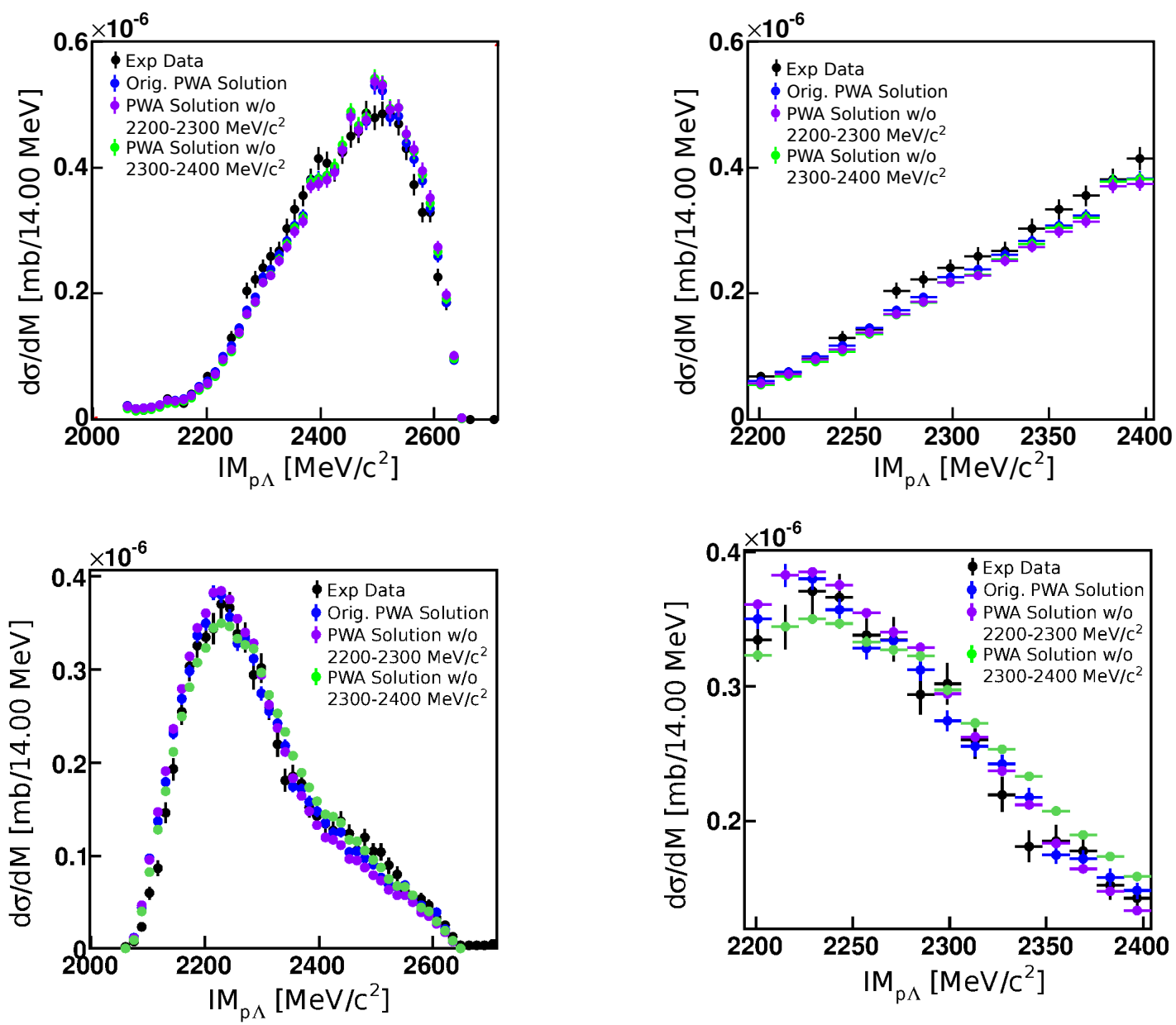

Figure 2: Invariant Mass of $p \Lambda$ for the two data sets (black points) shown with the best PWA solution, fitted to the complete HADES statistic (blue dots). Upper row: HADES data, lower row: WALL data. Compared to these results are the two cross checks where I) events were rejected from the fit with a mass range of 2200-2300 MeV/c ${ }^{2}$ (violet points) and II) where a mass range of 2300-2400 MeV/c (green points) was rejected from the PWA fit. The right panel shows a zoom into these mass regions.

potential content of a $\bar{K} N N$ inside the data does not bias their interpretation with help of the PWA.

\section{Is There a New Signal or Not?}

After this reassuring test, a statistical comparison between the PWA solution and the experimental data can be performed. The performed analysis is a test of the null hypothesis $\left(H_{0}\right)$ where a model that includes only background processes (PWA solution) is compared to the experimental data. A statistical comparison between the $H_{0}$-hypothesis and the experimental data was performed in order to see if there is some event yield that is unlikely to be described by the model. This could hint towards the necessity to include a further signal into the PWA solution. In the test the discrepancy between model and data is calculated with a Pearson- $\chi_{P}^{2}$ :

$$
\chi_{P}^{2}=\frac{\left(m_{i}-\lambda_{i}\right)^{2}}{\lambda_{i}}
$$


With $m_{i}$ and $\lambda_{i}$ the number of measured and expected events (according to the model) in the bin i, respectively. The discrepancy is calculated bin-wise so that a local $p_{0}$ distribution is obtained. The local $p_{0}$-value is derived from the $\chi_{P}^{2}$ value by an integration of the standard $\chi^{2}$ probability density function, with Ndf degrees-of-freedom, from the determined $\chi_{p}^{2}$ value of the data to infinity:

$$
\text { p-value }=\int_{\chi_{P, d}^{2}}^{\infty} P\left(\chi^{2}, N d f\right) d \chi^{2} .
$$

This calculation has been done separately for the four best solutions of the PWA variation and the two data sets (HADES and WALL). The result of the local $p_{0}$ calculation is shown in Figure 3. The spread between the four best solutions is expressed by the gray area. A small $p_{0}$-value indicates
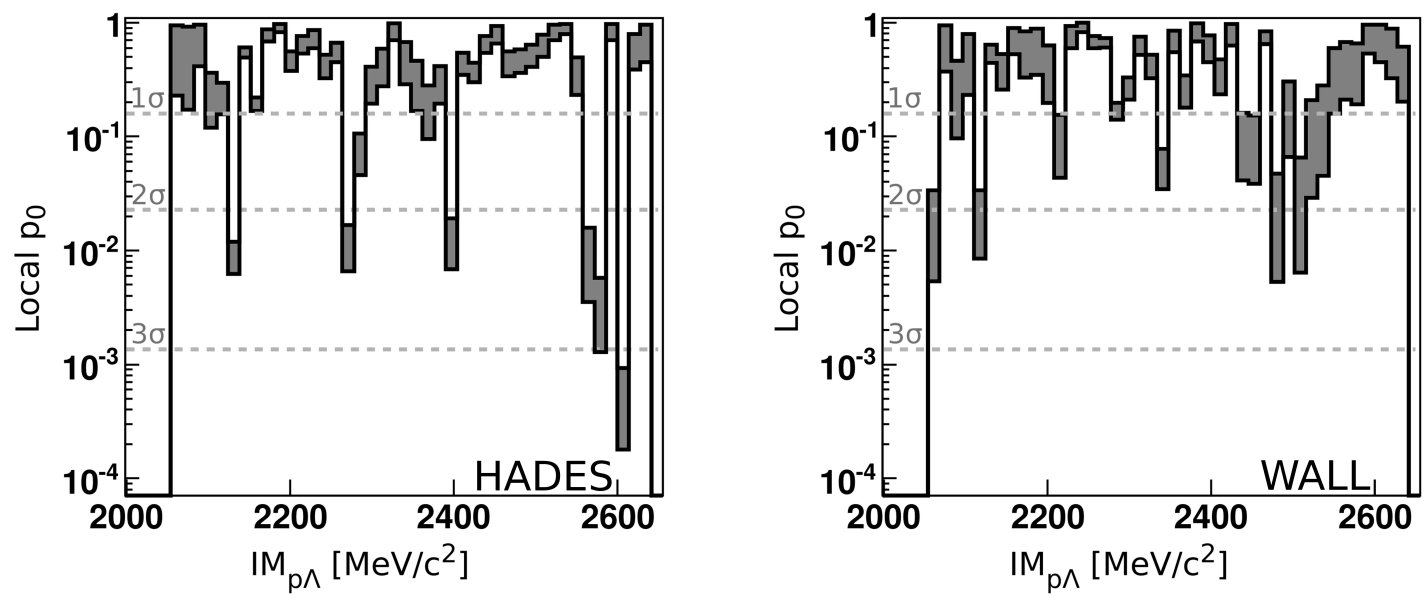

Figure 3: The local $\mathrm{p}_{0}$-value as a function of $\mathrm{IM}_{p, \Lambda}$, shown for both data sets individually. The grey spread is due to the systematic uncertainty and obtained from the four best solutions of the PWA fit. The horizontal dashed lines mark the discrepancy in terms of equivalent significance $(n \sigma)$.

a poor agreement between data and model. The smaller the $p_{0}$-value is, the lower is the probability that the deviation is caused by a random fluctuation, if the model is the true hypothesis. The gray-dashed horizontal lines express the deviation in terms of $n \sigma$, which is called equivalent significance and a common term in particle physics [49]. A deviation larger than $3 \sigma$ could, according to convention, hint to the presence of a new signal. Such a large deviation is only present in one data set (HADES) at a mass of $\mathrm{M}=2600 \mathrm{MeV} / \mathrm{c}^{2}$. It is not confirmed by the WALL data set and, furthermore it is a downward fluctuation of the data compared to the model (see Figure 1). Thus, as a result of this test no large deviation of the data from the null-hypothesis was found, making this model a good assumption for the production process. No hint of a new signal exceeding the standard contributions of $\mathrm{N}^{*}$ production and non-resonant $p \Lambda K^{+}$formation has been found in this analysis. As a consequence, only the determination of an upper limit remains which constrains a possible kaonic cluster production in $\mathrm{p}+\mathrm{p}$ reaction at a beam kinetic energy of $3.5 \mathrm{GeV}$.

\section{How to Obtain an Upper Limit}

The established PWA solutions offer the possibility to include an additional signal due to the production of a $\bar{K} N N$ in a consistent way. In this picture, the kaonic cluster can be produced out of 
three different initial proton+proton configurations:

$$
\begin{aligned}
& \text { WaveA: ' } p+p^{\prime} \quad\left({ }^{1} S_{0}\right) \rightarrow{ }^{\prime} p p K^{-}-K^{+} \quad\left({ }^{1} S_{0}\right), \\
& \text { WaveB: }{ }^{\prime} p+p^{\prime} \quad\left({ }^{3} P_{1}\right) \rightarrow{ }^{\prime} p p K^{-}-K^{+^{\prime}} \quad\left({ }^{1} P_{1}\right), \\
& \text { WaveC: }{ }^{\prime} p+p^{\prime} \quad\left({ }^{1} D_{2}\right) \rightarrow{ }^{\prime} p p K^{-}-K^{+^{\prime}} \quad\left({ }^{1} D_{2}\right) .
\end{aligned}
$$

Where, in the final state, the " $p p K^{-"}$ and the $K^{+}$have different angular momenta between each other. As interference effects are included, each wave with the production of a kaonic nuclear bound state can interfere different with the other waves within the same $J^{P}$ group. Therefore, each wave has to be tested separately within the model.

For the value of an upper limit the amplitude of the kaonic cluster production ( $a_{1}$ from Eq. (4.1)) was increased step-wise from zero $\left(H_{0}\right)$ to a value which produced a visible signal of the cluster, resulting in a new hypothesis $H_{\mu}$. The new hypothesis was tested against the data. The hypothesis was rejected as unlikely, as soon as the p-value of the test was lower than necessary for a $C L_{s}=95 \%$ confidence level [50-52]. Figure 4 illustrates the p-value as a function of the increasing amplitude strength. An inclusion of a new signal worsens the data description step-bystep, the higher the included signal strength is. The test was performed separately for Wave A, B, and C. Figure 4 shows an example of the inclusion of a kaonic cluster into one of the four best hypotheses $H_{0}$. The amplitude which delivers a p-value lower than indicated by the red-dashed line was rejected as improbable hypothesis and defines an upper limit on the production strength of a possible intermediate kaonic cluster state. This test is done for a number of hypothetical masses and widths and is subject of a forthcoming publication.
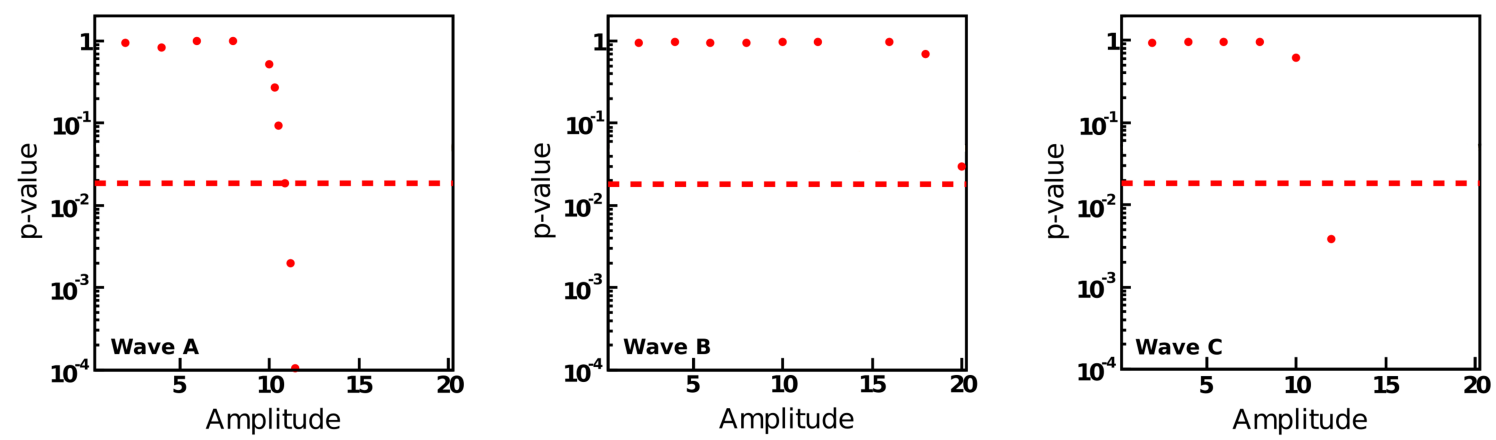

Figure 4: The p-value of the HADES data set as a function of the amplitude of the three kaonic cluster waves (A, B and C). The $\mathrm{CL}_{s}$ limit of $95 \%$ is shown by the red- dashed line.

\section{Conclusions}

Due to the presence of intermediate $\mathrm{N}^{*}$ resonances a model description of the $p K^{+} \Lambda$ final state is complex. The partial wave analysis, performed with the Bonn-Gatchina framework, is an elegant solution of this problem, which allowed us to derive a description of the measured event distributions. While we aim to describe background processes that do not contain a kaonic cluster signal, cross checks showed that the PWA result is not disturbed if possible signal events 
are included into the analysis. A statistical analysis of bin by bin fluctuations showed no significant deviation of the data from the PWA model that could be attributed to a new signal. We have, thus, continued to determine an upper limit on the production strength of the kaonic nuclear cluster in the reported reaction with help of the $C L_{s}$ method. The final result will be reported soon.

\section{Acknowledgments}

The HADES collaboration gratefully acknowledges the support by the grants LIP Coimbra, Coimbra (Portugal) PTDC/FIS/113339/2009, SIP JUC Cracow, Cracow6 (Poland): N N202 286038 28-JAN-2010 NN202198639 01-OCT-2010, HZ Dresden-Rossendorf (HZDR), Dresden (Germany) BMBF 06DR9059D, TU München, Garching (Germany) MLL München: DFG EClust 153, VH-NG-330 BMBF 06MT9156 TP5 GSI TMKrue 1012 NPI AS CR, Rez, Rez (Czech Republic) MSMT LC07050 GAASCR IAA100480803, USC - S. de Compostela, Santiago de Compostela (Spain) CPAN:CSD2007-00042, Goethe University, Frankfurt (Germany): HA216/EMMI HIC for FAIR (LOEWE) BMBF:06FY9100I GSI F\&E.

\section{References}

[1] Y. Nogami, Phys. Lett. 7, no. 4, 288 (1963).

[2] M. Krell, Phys. Rev. Lett. 26, 584 (1971).

[3] J. H. Koch, M. M. Sternheim and J. F. Walker, Phys. Rev. Lett. 26, 1465 (1971), Phys. Rev. C 5, 381 (1972).

[4] R. Seki, Phys. Rev. C 5, 1196 (1972).

[5] A. E. Kudryavtsev, V. D. Mur and V. S. Popov, JETP Lett. 37, 489 (1983) [Pisma Zh. Eksp. Teor. Fiz. 37, 412 (1983)], Phys. Lett. B 143, 41 (1984).

[6] V. S. Popov, A. E. Kudryavtsev, V. I. Lisin and V. D. Mur, Sov. Phys. JETP 61, 420 (1985) [Zh. Eksp. Teor. Fiz. 88, 715 (1985)].

[7] L. R. Staronski and S. Wycech, Czech. J. Phys. B 36, 903 (1986).

[8] S. Wycech, Nucl. Phys. A 450, 399C (1986).

[9] P. J. Fink, Jr., J. W. Schnick and R. H. Landau, Phys. Rev. C 42, 232 (1990).

[10] C. J. Batty, E. Friedman, A. Gal and G. Kaelbermann, Nucl. Phys. A 535, 548 (1991).

[11] A. Gal, E. Friedman and C. J. Batty, Nucl. Phys. A 606, 283 (1996).

[12] E. Friedman and A. Gal, Nucl. Phys. A 658, 345 (1999) [nucl-th/9907052].

[13] E. Friedman and A. Gal, Phys. Lett. B 459, 43 (1999) [nucl-th/9902036].

[14] A. Baca, C. Garcia-Recio and J. Nieves, Nucl. Phys. A 673, 335 (2000) [nucl-th/0001060].

[15] S. Hirenzaki, Y. Okumura, H. Toki, E. Oset and A. Ramos, Phys. Rev. C 61, 055205 (2000).

[16] T. Kishimoto, Phys. Rev. Lett. 83, 4701 (1999) [nucl-th/9910014].

[17] Y. Akaishi and T. Yamazaki, Nucl. Phys. A 684, 409 (2001).

[18] T. Yamazaki and Y. Akaishi, Phys. Lett. B 535, 70 (2002).

[19] Y. Akaishi and T. Yamazaki, Phys. Rev. C 65, 044005 (2002). 
[20] N. V. Shevchenko, A. Gal and J. Mares, Phys. Rev. Lett. 98, 082301 (2007) [nucl-th/0610022].

[21] N. V. Shevchenko, A. Gal, J. Mares and J. Revai, Phys. Rev. C 76, 044004 (2007).

[22] Y. Ikeda and T. Sato, Phys. Rev. C 76, 035203 (2007) [arXiv:0704.1978 [nucl-th]].

[23] Y. Ikeda and T. Sato, Phys. Rev. C 79, 035201 (2009) [arXiv:0809.1285 [nucl-th]].

[24] Y. Ikeda, H. Kamano and T. Sato, Prog. Theor. Phys. 124, 533 (2010) [arXiv:1004.4877 [nucl-th]].

[25] E. Oset, D. Jido, T. Sekihara, A. Martinez Torres, K. P. Khemchandani, M. Bayar and J. Yamagata-Sekihara, Nucl. Phys. A 881, 127 (2012) [arXiv:1203.4798 [hep-ph]].

[26] M. Bayar and E. Oset, arXiv:1207.1661 [hep-ph].

[27] M. Bayar and E. Oset, Nucl. Phys. A 883, 57 (2012) [arXiv:1203.5313 [nucl-th]].

[28] J. Revai and N. V. Shevchenko, arXiv:1403.0757 [nucl-th].

[29] T. Yamazaki and Y. Akaishi, Phys. Rev. C 76, 045201 (2007) [arXiv:0709.0630 [nucl-th]].

[30] A. Arai, M. Oka and S. Yasui, Prog. Theor. Phys. 119, 103 (2008) [arXiv:0705.3936 [nucl-th]].

[31] A. Dote, T. Hyodo and W. Weise, Nucl. Phys. A 804, 197 (2008) [arXiv:0802.0238 [nucl-th]].

[32] A. Dote, T. Hyodo and W. Weise, Phys. Rev. C 79, 014003 (2009) [arXiv:0806.4917 [nucl-th]].

[33] S. Wycech and A. M. Green, Phys. Rev. C 79, 014001 (2009) [arXiv:0808.3329 [nucl-th]].

[34] M. Faber, A. N. Ivanov, P. Kienle, J. Marton and M. Pitschmann, Int. J. Mod. Phys. E 20, 1477 (2011).

[35] N. Barnea, A. Gal and E. Z. Liverts, Phys. Lett. B 712, 132 (2012) [arXiv:1203.5234 [nucl-th]].

[36] A. Gal, arXiv:1011.6322 [nucl-th].

[37] A. Gal, Nucl. Phys. A 914, 270 (2013) [arXiv:1301.2145 [nucl-th]].

[38] M. Agnello et al. [FINUDA Collaboration], Phys. Rev. Lett. 94, 212303 (2005).

[39] G. Bendiscioli, A. Fontana, L. Lavezzi, A. Panzarasa, A. Rotondi and T. Bressani, Nucl. Phys. A 789, 222 (2007).

[40] G. Bendiscioli, T. Bressani, L. Lavezzi and P. Salvini, Eur. Phys. J. A 40, 11 (2009).

[41] T. Yamazaki, M. Maggiora, P. Kienle, K. Suzuki, A. Amoroso, M. Alexeev, F. Balestra and Y. Bedfer et al., Phys. Rev. Lett. 104, 132502 (2010) [arXiv:1002.3526 [nucl-ex]].

[42] M. Maggiora et al. [DISTO Collaboration], Nucl. Phys. A 835, 43 (2010) [arXiv:0912.5116 [hep-ex]].

[43] A. O. Tokiyasu et al. [LEPS Collaboration], Phys. Lett. B 728, 616 (2014).

[44] G. Agakishiev et al. [HADES Collaboration], Eur. Phys. J. A 41, 243 (2009).

[45] E. Epple et al. [HADES Collaboration], PoS BORMIO 2012, 016 (2012).

[46] L. Fabbietti et al. [HADES Collaboration], Nucl. Phys. A 914, 60 (2013) [arXiv:1305.2631 [nucl-ex]].

[47] A. V. Anisovich and A. V. Sarantsev, Eur. Phys. J. A 30, 427 (2006) [hep-ph/0605135].

[48] A. Anisovich, V. Anisovich, E. Klempt, V. Nikonov and A. Sarantsev, Eur. Phys. J. A 34, 129 (2007).

[49] J. Beringer et al. [Particle Data Group Collaboration], Phys. Rev. D 86, pp. 394 (2012).

[50] T. Junk, Nucl. Instrum. Meth. A 434, 435 (1999) [hep-ex/9902006].

[51] A. L. Read, In *Geneva 2000, Confidence limits* 81-101.

[52] A. L. Read, J. Phys. G 28, 2693 (2002). 